\title{
Increased Transmitter Release at Excitatory Synapses Produced by Direct Activation of Adenylate Cyclase in Rat Hippocampal Slices
}

\author{
Laura E. Chavez-Noriega and Charles F. Stevens \\ Howard Hughes Medical Institute, Salk Institute, La Jolla, California 92037
}

The field EPSP recorded in the CA1 region of rat hippocampal slices is potentiated by bath application of the direct adenylate cyclase activator forskolin (Chavez-Noriega and Stevens, 1992a). We have now used the whole-cell patchclamp technique to analyze the effect of forskolin on evoked synaptic currents and on spontaneous and miniature excitatory postsynaptic currents (sEPSCs and mEPSCs) recorded in rat hippocampal slices in order to determine the relative contributions of pre- and postsynaptic mechanisms to this increased synaptic strength.

Application of $50 \mu \mathrm{M}$ forskolin in the presence of 3-isobutyl1-methylxanthine (IBMX; a phosphodiesterase inhibitor) enhanced the evoked EPSC (eEPSC) peak amplitude to $230 \pm$ $43 \%$ of control $(n=13)$. No significant change in sEPSC or in mEPSC amplitude was detected after forskolin addition (106 $\pm 7 \%, n=9$ ), indicating that postsynaptic receptor sensitivity at synaptic junctions is not greatly affected. In contrast, a large increase in SEPSC and mEPSC frequency was noted in all cells $(299 \pm 81 \%)$.

Following forskolin application, the amplitude distribution of evoked synaptic currents shifted to larger values, but more significantly, a sharp decrease in failure rate was produced in all cells tested. Also, a significant correlation was found between the potentiation produced by forskolin in IBMX on the eEPSC and the ratio of the squared coefficient of variation (CV $=\mathrm{SD} /$ mean). Finally, a quantal analysis of four cells was consistent with the hypothesis that transmitter release was increased by forskolin/IBMX with, if anything, a concomitant decrease in quantal size. Together, these observations indicate that presynaptic mechanisms significantly contribute to the enhancement produced by this diterpene.

Altogether, our data indicate that activation of the CAMP cascade can enhance evoked and spontaneous release of neurotransmitter in Schaffer/commissural terminals in field CA1 of rat hippocampal slices.

[Key words: CA1, excitatory synapses, pyramidal neurons, transmitter release, presynaptic mechanisms, glutamatergic synapses, protein kinases, adenylate cyclase, cAMP, forskolin, 3-isobutyl-1-methylxanthine]

\footnotetext{
Received Mar. 25, 1993; revised July 1, 1993; accepted July 6, 1993.

This work was supported by Howard Hughes Medical Institute and NIH Grant 5-ROI NS 12961. Postdoctoral Fellowship S-60-92 to L.E.C.-N. from the American Cancer Society (California Division) also provided support for the final stages of this project.

Correspondence should be addressed to Dr. Charles F. Stevens, Salk Institute, 10010 North Torrey Pines Road, La Jolla, CA 92037

Copyright C 1994 Society for Neuroscience $0270-6474 / 94 / 140310-08 \$ 05.00 / 0$
}

A substantial body of evidence implicates kinase action in the production and maintenance of long-term potentiation (LTP; Bliss and Collingridge, 1993). For example, kinase inhibitors prevent the induction of LTP, and have even been reported to inhibit the expression of LTP once it is established and to block the conversion of LTP to a more stable form. Also, the concentration of cAMP (the activator of cAMP-dependent protein kinase, PKA) increases under conditions that produce LTP (Stanton and Sarvey, 1985a), and these increases are suppressed by the NMDA receptor blocker aminophosphonovalerate (Chetkovitch et al., 1991), a drug known also to prevent LTP induction (Bliss and Collingridge, 1993).

Because of the possible role for kinases in LTP, we have been interested in determining the mechanisms through which the kinases might modify synaptic strength. In earlier work we have found that agents acting on the CAMP/PKA cascade have a profound effect on synaptic transmission: the combination of forskolin (an activator of adenylate cyclase, the enzyme that produces cAMP; Daly et al., 1982; Onodera and Kogure, 1989) and 3-isobutyl-1-methylxanthine (IBMX; an inhibitor of the phosphodiesterase involved in the destruction of cAMP; Smellie et al., 1979; Phillis and Wu, 1981) greatly increases synaptic strength. These experiments, however, used field potential recordings, so we were unable to determine whether the pharmacological actions we observed were pre- or postsynaptic (Chavez-Noriega and Stevens, 1992a). A postsynaptic role is supported by work carried out in cultured hippocampal neurons in our laboratory (Greengard et al., 1991) and others (Wang et al., 1991, 1993), but the role of a possible presynaptic action has not been investigated. We have therefore undertaken the experiments described here to determine what fraction of the forskolin/IBMX effect on synaptic strength occurs through an increase in transmitter release as opposed to enhancement of the postsynaptic response to neurotransmitter.

We have used the whole-cell recording technique to confirm that forskolin/IBMX increases synaptic strength in the CAI region of hippocampal slices. Quantal analysis of synaptic currents reveals that the major effect of these agents is on transmitter release, a conclusion confirmed by the observation that miniature excitatory postsynaptic currents (mEPSCs) are much less altered in size by forskolin/IBMX than are the corresponding evoked EPSCs (eEPSCs).

Preliminary data have been reported in abstract form (Chavez-Noriega and Stevens, 1992b).

\section{Materials and Methods}

Transverse hippocampal slices approximately $350-400 \mu \mathrm{m}$ thick were prepared from young (9-22 d old) Sprague-Dawley rats. The animals were anesthetized with sodium pentobarbital $(40 \mathrm{mg} / \mathrm{kg}$, i.p.); the brain 
was quickly dissected and sliced using a vibratome (Campden Instruments). The Krebs' solution used for these procedures contained sucrose (240 mM) substituting for $\mathrm{NaCl}$ (see below; Aghajanian and Rasmussen, 1989). A surgical cut was made to isolate area CA1 from CA3. Slices were kept in an interface chamber at room temperature for at least 1 $\mathrm{hr}$ before recording. In the recording chamber, slices were fully submerged and continuously perfused $(1-2 \mathrm{ml} / \mathrm{min}$ ) with Krebs' solution of the following composition (in $\mathrm{mM}$ ): $\mathrm{NaCl}, 120 ; \mathrm{KCl}, 3 ; \mathrm{MgCl}_{2}, 1$; $\mathrm{NaH}_{2} \mathrm{PO}_{4}, 1.2 ;$ D-glucose, $11 ; \mathrm{NaHCO}_{3}, 23 ; \mathrm{CaCl}_{2}, 2$ (290 mOsm); saturated with $95 \% \mathrm{O}_{2}, 5 \% \mathrm{CO}$ to maintain a $\mathrm{pH}$ of 7.4 . Temperature was maintained at $32 \pm 1^{\circ} \mathrm{C}$, except for about one-fifth of the experiments, which were performed at room temperature. No difference was noted between these two groups.

Tight-seal (1-10 G $\Omega$ ), whole-cell voltage-clamp recordings were obtained from neurons in the CAl pyramidal cell body layer. Patch electrodes were pulled from borosilicate glass (Drummond Scientific Co., Broomall, PA) and had a resistance of 3-6 M $\Omega$ when filled with a solution containing (in mM) potassium gluconate, $130 ; \mathrm{KCl}, 5 ; \mathrm{EGTA}, 0.5 ; \mathrm{MgCl}_{2}$, 2; HEPES, 20; ATP-Mg, 2; and GTP-Li, 0.3 (290 mOsm, pH adjusted to 7.3 with $\mathrm{KOH}$ ). An Axopatch 200 amplifier (Axon Instruments), with compensation for series resistance, was used for whole-cell recording. A bipolar tungsten stimulating electrode (F.H.C., Brunswick, ME) placed in stratum radiatum was used to activate the recorded neurons synaptically. One hundred microsecond square pulses were delivered at $0.2-1 \mathrm{~Hz}$ using a stimulus isolator (World Precision Instruments A 350D). All experiments were performed in the presence of $20 \mu \mathrm{M}$ bicuculline

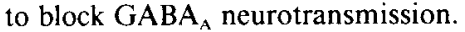

Data were filtered at $1 \mathrm{kHz}$, amplified, and digitized at $2.5-10 \mathrm{kHz}$. Acquisition and analysis were carried out using programs written by $\mathrm{C}$. F. Stevens (AXOBASIC software, Axon Instruments).

To estimate the effect on the eEPSC peak amplitude and synaptic failure rate of forskolin in IBMX, the synaptic test stimulus intensity was lowered after IBMX addition in 6 of 13 experiments to ensure that synaptic transmission failures (cases in which a synaptic test stimulus did not produce a detectable EPSC) were evident before forskolin application. Mean amplitudes of eEPSCs were estimated taking into account all responses obtained within a given stable recording period, including synaptic transmission failures.

Data are expressed as mean \pm standard error of the mean $(X \pm$ SEM). The nonparametric, one-tailed Mann-Whitney $U$ test was used to assess statistical differences between experimental groups.

All drugs were bath applied from stock solutions. Bicuculline methochloride was purchased from Cambridge Research Biochcmicals (Wilmington, DE). Tetrodotoxin (TTX), forskolin, and 3-isobutyl-1-methylxanthine (IBMX) were obtained from Sigma (St. Louis, MO). I-(5-isoquinolinylsulfonyl)-2-methylpiperazine $(\mathrm{H}-7)$ was purchased from Biomol (Plymouth, PA). Drugs were dissolved in distilled water or Krebs. Forskolin and IBMX were dissolved in dimethyl sulfoxide (DMSO); final concentrations of DMSO in Krebs ranged from $0.05 \%$ to $0.1 \%$.

\section{Results}

Stable whole-cell patch recordings were obtained from $38 \mathrm{CA} 1$ pyramidal neurons. Membrane potential was maintained at -70 or $-60 \mathrm{mV}$. All cells included in this report showed no significant changes $(<15 \%)$ in input or in series resistance during the recording period. Those few neurons displaying larger changes were rejected for further analysis.

\section{Forskolin enhances the eEPSCs recorded from voltage- clamped CAI pyramidal neurons}

The basic effect we wished to examine, the increase in synaptic strength produced by forskolin in the presence of IBMX (forskolin/IBMX) is illustrated in Figure 1.

Bath application of $50 \mu \mathrm{M}$ forskolin produced a modest increase in the eEPSC peak amplitude, to $127 \pm 14 \%$ of control ( $n=9$; Fig. 2). IBMX itself $(50 \mu \mathrm{M})$ also enhanced the eEPSC amplitudes to $166 \pm 15 \%(n=10$; Fig. 2$)$ of the control value. In the presence of IBMX, however, forskolin enhanced the eEPSC amplitude to $230 \pm 43 \%$ of control $(n=13$; Figs. 1, 2). A new

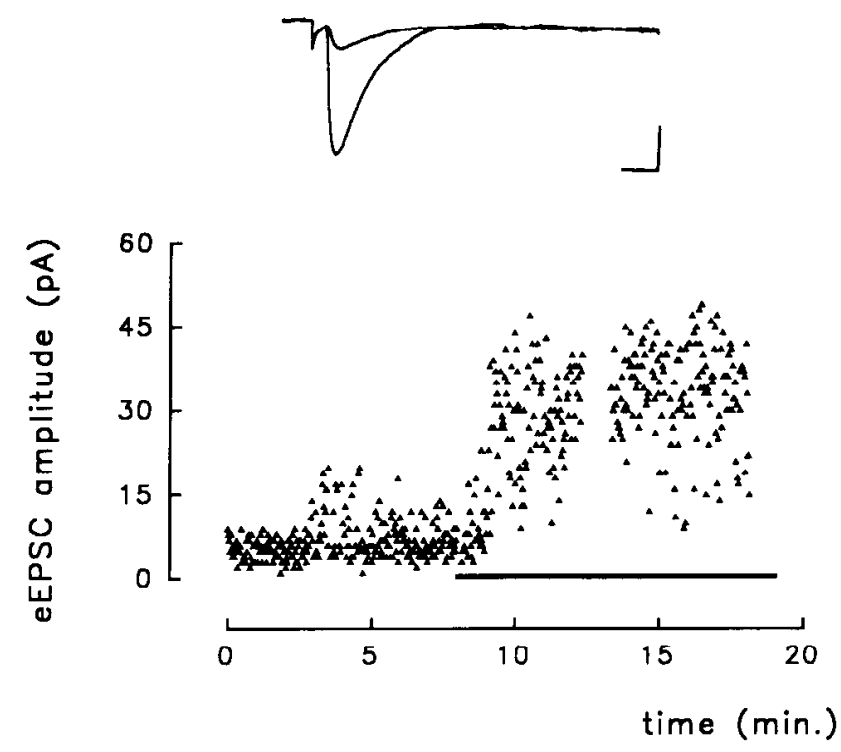

Figure 1. Time course of the action of forskolin on the peak amplitude of evoked synaptic currents. Each data point represents a single response evoked before and during the application of $50 \mu \mathrm{M}$ forskolin (horizontal bar) in a CA1 pyramidal cell. IBMX and bicuculline were present throughout the recording. Membrane holding potential was $-70 \mathrm{mV}$. The superimposed traces above illustrate responses evoked before and after forskolin application. Each is the average of 30-40 individual responses (the stimulus artifact has been blanked for clarity). Calibration: $10 \mathrm{pA}, 8 \mathrm{msec}$.

baseline was obtained after IBMX addition, so the potentiation indicated is that in excess of any produced by IBMX (see Fig. 1). The reason for reducing the synaptic test stimulus intensity (SI) and obtaining a new baseline was to adjust the SI such that in about half of the trials a failure in synaptic transmission would occur (see Materials and Methods) before forskolin was added.

The enhancement produced by forskolin under these conditions was evident within about $2-3 \mathrm{~min}$ of its addition to the bath solution (Fig. 1). The potentiation produced by forskolin in IBMX was significantly larger than in control medium $(p=$ 0.025 ). Washout to drug-free Krebs resulted in a reversal of a large proportion $(68 \pm 7 \%)$ of the enhancement produced by IBMX and forskolin (following 30-40 min wash, $n=4$ cells; data not shown).

We have found that the increase produced on the field EPSP slope by forskolin in IBMX is most likely mediated via the activation of adenylate cyclase, since the effect is not reproduced by the inactive forskolin analog 1,9-dideoxyforskolin (Hoshi et al., 1988; Wagoner and Pallotta, 1988), and is suppressed by the adenylate cyclase inhibitors (Haslam et al., 1978) SQ-22536 and $2^{\prime}, 5^{\prime}$-dideoxyadenosine (Chavez-Noriega and Stevens, 1992a). In a preliminary group of experiments, we tested the effect of $\mathrm{H}-7$, a potent protein kinase inhibitor (Hidaka et al., 1984), on the enhancement of the eEPSC amplitude produced by forskolin. In the presence of $\mathrm{H}-7$ (300 $\mu \mathrm{M}$, applied 30-90 min preceding the application of forskolin; IBMX and bicuculline also present), the effect of forskolin was suppressed: the mean eEPSC amplitude after forskolin application was $94 \pm$ $26 \%(n=3)$; a small increase (to $131 \%$ ) was observed only in one cell. The enhancement produced by forskolin under these conditions was significantly smaller than in the absence of the kinase inhibitor ( $p<0.05$; Fig. 2).

The effect of bath-applied cAMP and/or its membrane-per- 

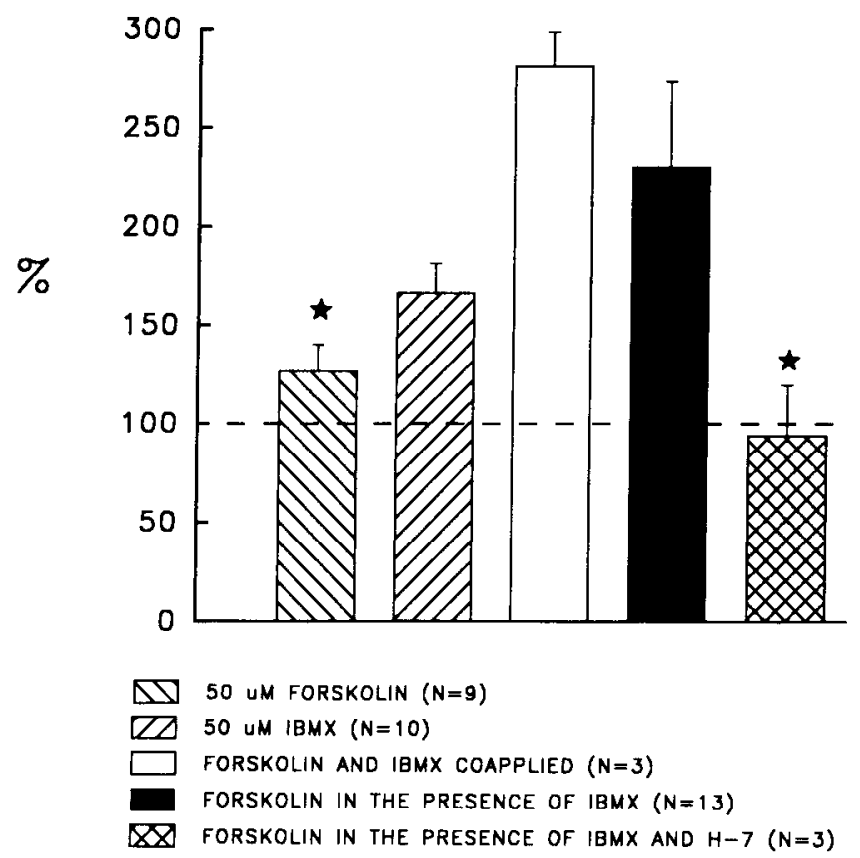

Figure 2. Mean (+SEM) change in the eEPSC peak amplitude produced by forskolin in the absence and presence of IBMX. Notice that coapplication of IBMX and forskolin produces an increase about twice as large as the arithmetic sum of the effect of either drug on its own. The potentiation produced by forskolin in the presence of IBMX (solid har) was calculated after a stable baseline was obtained following IBMX application; this was statistically larger than the increase produced in control medium $(p<0.05$, star). The effect of forskolin in IBMX when $\mathrm{H}-7$ was present was significantly smaller than its control group $(p<$ 0.05, star).

meable analogs was not studied because they depress synaptic transmission and inhibit cAMP accumulation via the activation of extracellular adenosine $A_{1}$ receptors (Dunwiddie and Hoffer, 1980; Dunwiddie and Fredholm, 1989), complicating the interpretation of the action of these compounds.

Since the action of forskolin was most conspicuous in the presence of IBMX, all subsequent experiments were carried out in the presence of this methylxanthine (referred to as forskolin/ IBMX).

\section{Forskolin does not significantly increase the amplitude of} sEPSCs and MEPSCs; their frequency, however, is increased

Because earlier investigators had reported that forskolin acts postsynaptically to increase the amplitude of mEPSCs (Greengard et al., 1991; Wang et al., 1991), our first hypothesis to explain the large increase in eEPSC amplitude was that forskolin/IBMX causes a modification of channel properties that resulted in larger postsynaptic currents. The effects of forskolin on mEPSC amplitude in culture were smaller than the approximately twofold increase of synaptic strength we observed in slices. Nevertheless, Greengard et al. (1991) reported modifications of single-channel properties in patches treated with the catalytic subunit of PKA that are large enough potentially to account for our effect. To estimate the contribution of increased eEPSC amplitude produced by forskolin/IBMX, then, we monitored sEPSCs in cells for which eEPSCs were also recorded.

None of the four cells analyzed displayed an increase above $11 \%$ in the mean sEPSC amplitude $(99.5 \pm 6 \%)$ following forskolin application in IBMX. The sEPSC amplitude distributions
A

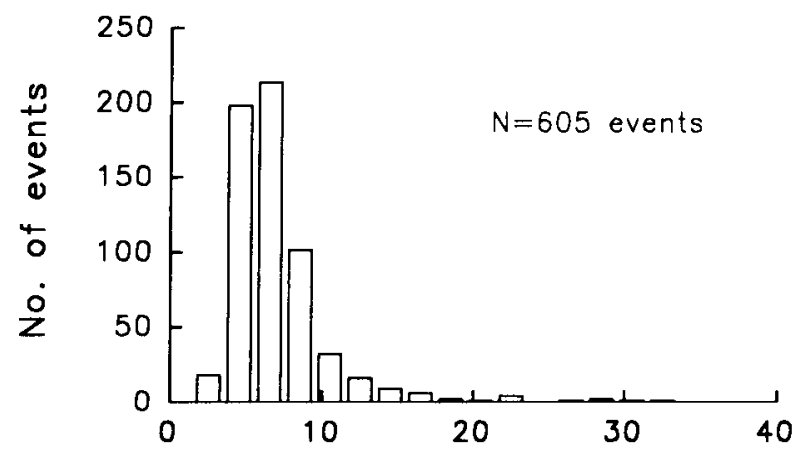

B

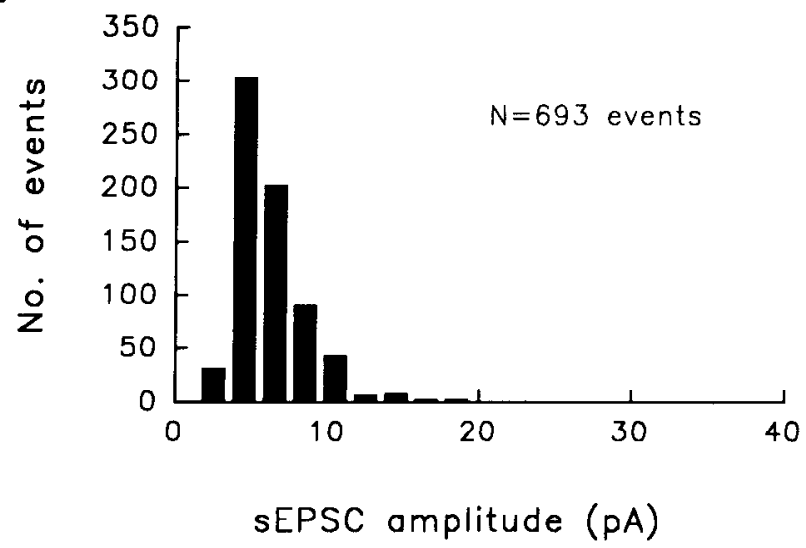

Figure 3. sEPSC amplitude distributions before and following forskolin application. The detection threshold for SEPSCs was set at two times the SD of the background noise (the smallest detectable events ranged from 3 to $5 \mathrm{pA}$ in all cells). Shown are histograms obtained from one representative neuron about $12 \mathrm{~min}$ before $(A)$ and about $10-15 \mathrm{~min}$ after forskolin addition $(B)$ in the presence of IBMX. Mean sEPSC amplitudes were 7.0 and $6.2 \mathrm{pA}$ before and after forskolin, respectively.

were basically unchanged by the drug treatment (Fig. 3). This is in contrast to the changes in the eEPSC for these same four cells, which ranged from $109 \%$ to $624 \%$ (284 $\pm 116 \%$; compare Figs. 3, 5). An increase in sEPSC frequency was noted, with post-forskolin values ranging from $163 \%$ to $452 \%(257 \pm 68 \%$, $n=4)$

The interpretation of sEPSC amplitude is complicated by the fact that these events represent a mixture of miniature and evoked (by spontaneous cell firing) synaptic currents. We therefore studied another group of neurons in which the effect of forskolin/ IBMX was examined in the presence of TTX; events in this situation should be exclusively mEPSCs. We found that application of forskolin enhanced the amplitude of mEPSCs above $25 \%$ in only two of five cells recorded (average $-137 \%$ ), but overall (111 $\pm 12 \%, n=5)$, no significant increase in mEPSC amplitude was detected (Fig. 4). A clear increase in mEPSC frequency was observed in all cells $(331 \pm 139)$, similar to our observations with sEPSCs.

It is conceivable that the mEPSCs may "run down" with time if, for example, the postsynaptic sensitivity to glutamate decays as a function of time after the whole-cell patch is established (Wang et al., 1991). The lack of enhancement may rather reflect a blockade of a reduction in amplitude when no activator of the cAMP cascade is supplied. To test for this, we monitored mEPSCs (in IBMX and bicuculline, as usual) before and after 

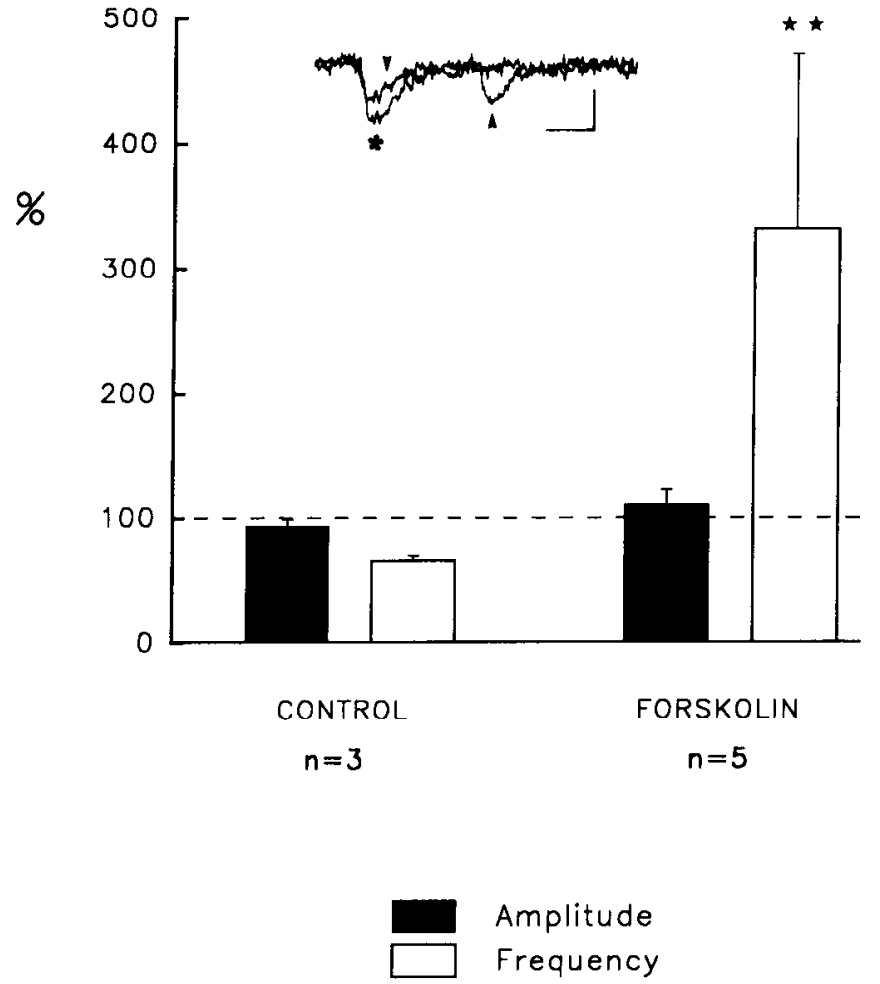

Figure 4. Mean (+SEM) changes in mEPSC amplitude and frequency in control cells and in neurons treated with forskolin. Forskolin did not significantly affect the mEPSC amplitude. In contrast, the mEPSC frequency was significantly increased. This was statistically larger than the changes recorded in the absence of forskolin addition $(p<0.02$, double stars). No significant "rundown" was detected in the mEPSC amplitude as a function of recording time (no forskolin added), although some decay in frequency was noted. Above, two superimposed single traces illustrating a miniature event before (asterisk), and a couple of them appearing in succession, after forskolin addition (arrowheads). Membrane holding potential, $-70 \mathrm{mV}$. Calibration: $10 \mathrm{pA}, 15 \mathrm{msec}$.

an interval similar to that left in the forskolin experiments. No significant change was found in the mEPSC amplitude (94 \pm $5 \%, n=3$; Fig. 4); the mEPSC frequency, however, scemed to be somewhat lower $(66 \pm 4 \%)$, in clear contrast to the changes seen in cells where forskolin was applied.

These data do not support a significant role for postsynaptic mechanisms in the increased synaptic strength produced by forskolin/IBMX under the conditions of our experiments.

\section{Quantal analysis supports a presynaptic mechanism of action for forskolin/IBMX}

Since we cannot account for the increased synaptic strength we have observed by postsynaptic mechanisms, we evaluated the hypothesis that forskolin/IBMX acts by increasing transmitter release. If transmitter release is increased by a presynaptic mechanism (either an increase in release probability or in the number of release sites), the number of stimuli that produce no detectable synaptic current ("failures") should decrease, as should the cocfficient of variation ( $\mathrm{CV}=\mathrm{SD} /$ mean) (Del Castillo and Katz, 1954; Bekkers and Stevens, 1990; Malinow and Tsien, 1990). Furthermore, a quantal analysis should (see Prince and Stevens, 1992) yield an increased estimate for the release parameter $m$, without a significant increase in the estimated quantal size $a$, as described below.

We have measured the distribution of the eEPSC amplitudes
A

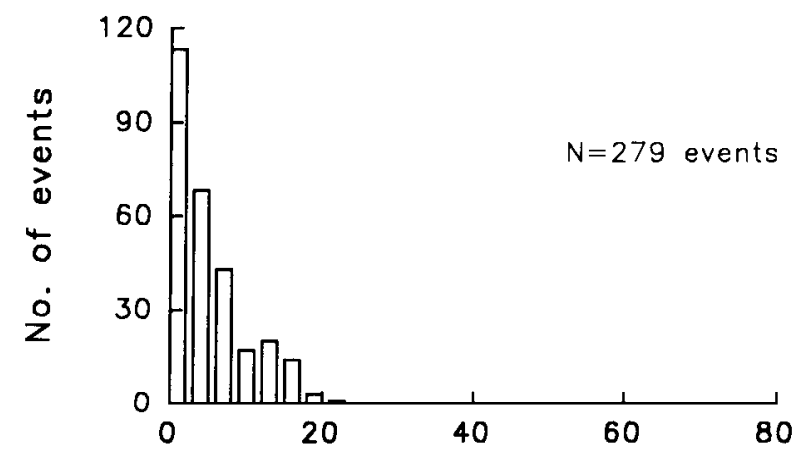

B

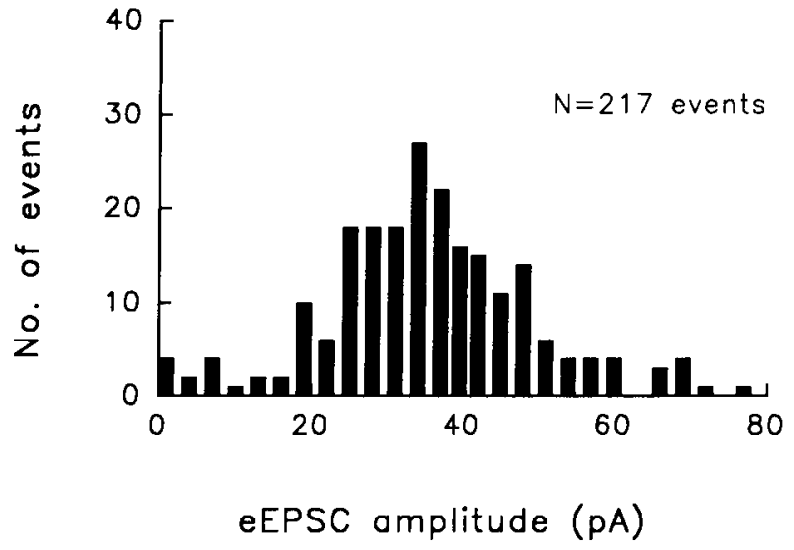

Figure 5. Amplitude distributions of evoked synaptic currents before and after forskolin addition: histograms of peak eEPSC amplitudes recorded in one cell during the $6 \mathrm{~min}$ period preceding $(A)$ and 3-7 min and 17-20 min following the application of forskolin $(B$; sEPSCs were recorded 8-16 min after forskolin addition). Mean eEPSC amplitudes were 4.7 and $35.5 \mathrm{pA}$ before and after forskolin, respectively. Membrane holding potential, $-70 \mathrm{mV}$. Data from the same cell illustrated in Figure 3 .

obtained before and after forskolin application in six cells (see Materials and Methods). We find that forskolin/IBMX not only produces the large increase in the avcrage size of eEPSCs described earlier, but also, as seen in Figure 5, causes a sharp decline in the fraction of stimuli that result in release failures. A decrease in failure rate (number of failures/total number of trials) was evident in all those cells that showed an increase in the eEPSC amplitude ( 5 of 6 cells tested) following forskolin application. The failure rate $(0.34 \pm 0.03)$ fell by $90 \%$, to a mean value of $0.03 \pm 0.02(n=5)$ in forskolin/IBMX. The sixth cell, which exhibited little change in the eEPSC after forskolin addition $(109 \%$, compared with the mean increase in the other five cells, $344 \pm 87$ ), showed a decrease of only $29 \%$ in failure rate. This suggests that forskolin/IBMX increases the likelihood of transmitter release or the number of release sites.

Changes in the coefficient of variation ( $\mathrm{CV}=\mathrm{SD} /$ mean) are unlikely to occur by manipulations that only affect the mean quantal amplitude. We found, however, that forskolin/IBMX consistently causes a decrease in the $\mathrm{CV}$ : a significant corrclation ( $p<0.05, n=13$ ) was observed between the changes in the mean eEPSC amplitude after forskolin addition and the ratio of the coefficient of variation squared (Fig. 6). Again, this observation supports the hypothesis that forskolin/IBMX acts presynaptically, increasing either the probability of transmitter release or the number of available release sites, or both. 


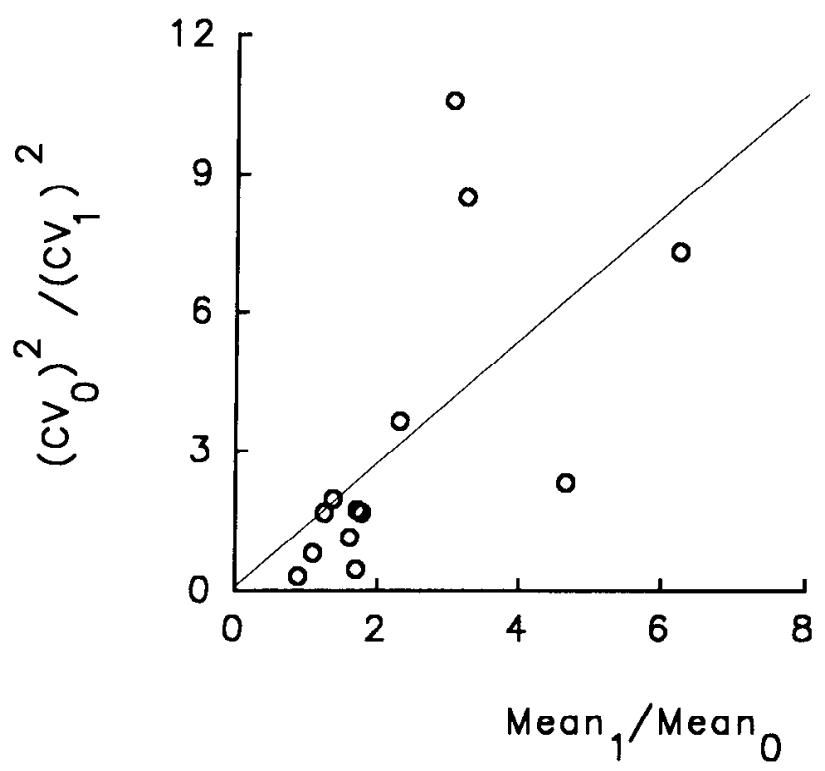

Figure 6. Plot displaying the ratio of the squared coefficient of variation as a function of the change in the eEPSC peak amplitude observed upon addition of forskolin in the presence of IBMX. The abscissa displays the ratio of the mean eEPSC peak amplitude recorded after $(I)$ and before $(\theta)$ forskolin addition in each cell; $\mathrm{CV}=\mathrm{SD} / \mathrm{mean}$ eEPSC amplitude. The regression line has been fitted (least squares approximation) through the data. Each symbol represents one cell $(n=13)$. The data points with $x$-values around 1 (mean $/$ mean $\left._{0} \leq 1\right)$ correspond to those two cells in which no enhancement was observed upon addition of forskolin. The regression coefficient is $0.62(p<0.05)$.

Because the amplitudes of individual quanta vary a lot for central synapses (Bekkers et al., 1990; Manabe et al., 1992; Raastad et al., 1992), one must take this quantal variability into account in using the Katz theory to describe fluctuations in eEPSC amplitudes. We have assumed that mEPSCs vary according to the distribution described earlier by Bekkers et al. (1990), and have fitted the experimental data to amplitude distributions predicted for Poisson release. Specifically, the probability $f(x)$ of an mEPSC amplitude $x$ is assumed to be approximated by the equation (see Bekkers et al., 1990)

$$
f(x)=\frac{1}{3 \sqrt{2 \pi b} x^{2 / 3}} e^{-\frac{\left(x^{1}-a\right)^{2}}{2 b}},
$$

where $a$ is the mean quantal amplitude and $b$ is a constant that determines the variance of quantal amplitudes; $b$ is selected to agree with the observed value of about 0.48 . The probability $p(k)$ that $k$ quanta will be released is assumed to be given by the Poisson distribution (Katz, 1969)

$$
p(k)=\frac{m^{k}}{k !} e^{-m}
$$

where $m$ is the Poisson parameter that determines the mean number of quanta released per stimulus. The predicted EPSC amplitude distribution $h(x)$ for an amplitude $x$ is then calculated from the equation

$$
h(x)=\sum_{k} p(k) f_{k}(x)
$$

where the function $f_{k}(x)$ is found from the equations
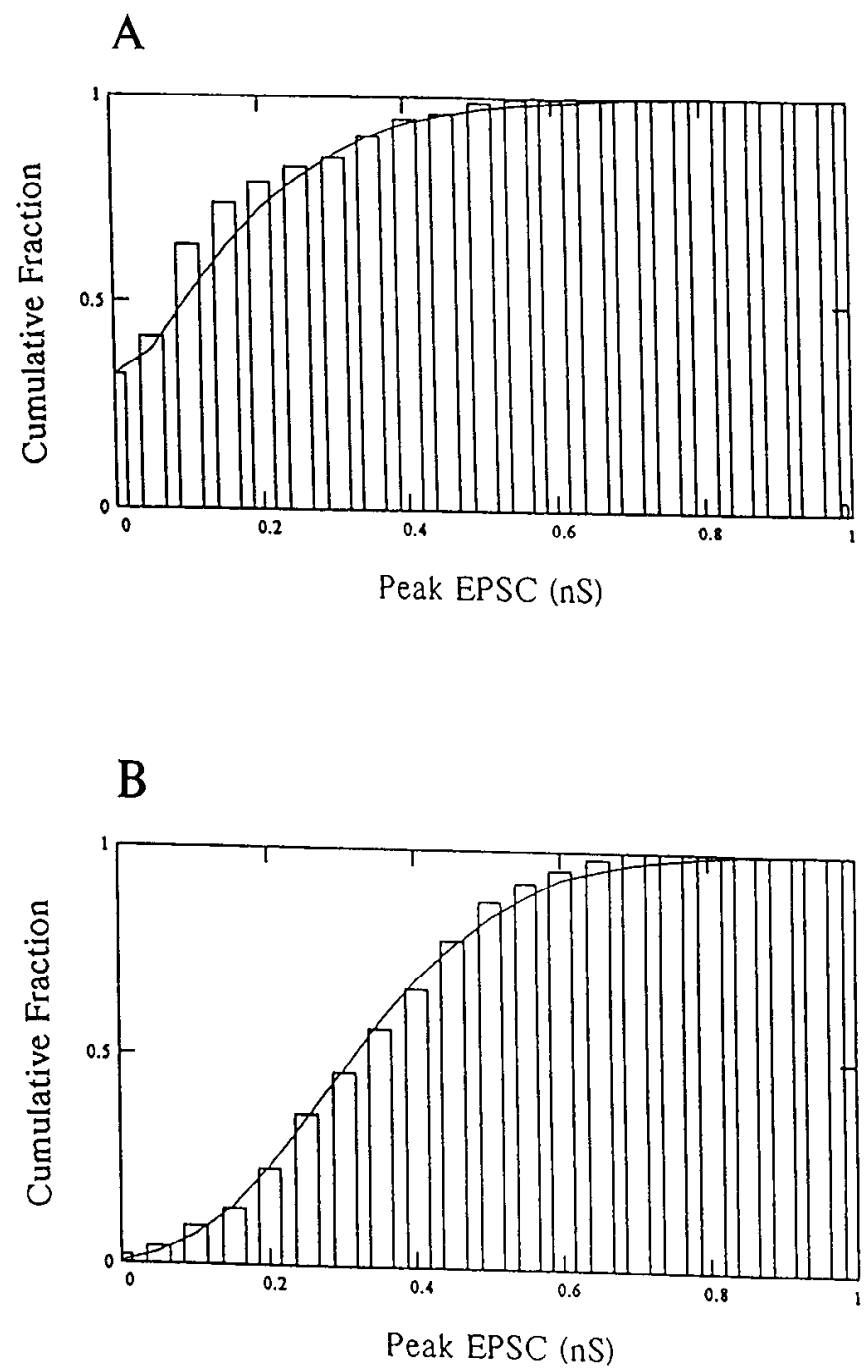

Figure 7. Cumulative probability of eEPSC peak conductance before $(A)$ and after $(B)$ forskolin addition (in IBMX) for a typical cell. The smooth curves are the predictions of the Katz theory, modified as described in the Results, with $m=1.123$ and $a=0.129 \mathrm{nS}$ before application of forskolin and $m=5$ and $a=0.066 \mathrm{nS}$ after forskolin was applied.

$$
\begin{aligned}
f_{1}(x) & =f(x) \\
f_{k+1}(x) & =\int_{0}^{x} f(\xi) f_{k}(x-\xi) d \xi .
\end{aligned}
$$

The parameters $a$ and $m$ were varied until the best fit between $h(x)$ and the observed amplitude distribution was achieved.

This procedure was carried out for four cells that had yielded a sufficient quantity of stationary data. In each of the four cases, the best fitting $a$ was little changed by the forskolin/IBMX treatment; in all four cases, drug treatment produced increases in the Poisson parameter $m$ with a 5.5-fold average increase (range 1.4-11.5). A typical example that compares predicted and observed eEPSC amplitude distributions is shown in Figure 7 . The best-fitting values of $a$ were, in all cases, within a factor of 2 of the observed average sEPSC amplitude for the cells in question. Comparison of the value for $a$ with mean sEPSC amplitude can be only semiquantitative because the distribution of synapses 
producing synaptic currents is unknown and cable attenuation can significantly affect amplitude.

We conclude, then, that the forskolin/IBMX treatment produces a large increase in synaptic strength primarily by augmenting transmitter release. If release probability $(p)$ is high enough to require a binomial amplitude distribution, both the release probability and the number of release sites $(N)$ can be estimated. But in the Poisson limit (whenever release probability is low), then, $N$ and $p$ always appear as a product $N p$, and one cannot distinguish changes in $N$ from changes in $p$. Because our amplitude distributions appeared to fall at the Poisson limit, we cannot decide if our increased transmitter release resulted from changes in release probability $(p)$ or in number of release sites $(N)$ or both.

\section{Discussion}

Our data indicate that presynaptic mechanisms are the main contributors to the increase in synaptic efficacy produced by the direct adenylate cyclase activator forskolin in field CAl of rat hippocampal slices.

The potentiation of synaptic strength produced by forskolin/ IBMX appears to be produced by activation of the cAMP transduction pathway, since (1) previous work had shown that the potentiation of the field EPSP elicited by this diterpene is not mimicked by the inactive forskolin analog 1,9-dideoxyforskolin, (2) it is blocked by adenylate cyclase inhibitors (Chavez-Noriega and Stevens, 1992a), and (3) preliminary data indicate that the PKA inhibitor $\mathrm{H}-7$ suppresses the enhancement produced by forskolin/IBMX on the eEPSC. Although $\mathrm{H}-7$ is a nonspecific protein kinase inhibitor (Hidaka et al., 1984), forskolin has not been found to interact with protein kinases (including protein kinase C), or phosphodiesterases (Seamon and Daly, 1986; Laurenza et al., 1989), suggesting that the most likely effect of $\mathrm{H}-7$ is on the enhanced cAMP cascade.

Application of forskolin in the absence of IBMX produced only a small increase in the eEPSC size. This observation correlates with the action of forskolin on the field EPSP (ChavezNoriega and Stevens, 1992a). Only in the presence of IBMX was a large increase revealed. At the concentration used, IBMX can also block adenosine receptors (Smellie et al., 1979; Phillis and $\mathrm{Wu}, 1981$ ), and tonic activation of $A_{1}$ receptors depresses synaptic transmission in field $\mathrm{CAl}$ and inhibits hippocampal adenylate cyclase activity (Dunwiddie and Hoffer, 1980; Dunwiddie and Fredholm, 1989). IBMX itself enhanced the field EPSP slope (Chavez-Noriega and Stevens, 1992a) and the eEPSC amplitude (in agreement with Prince and Stevens, 1992), and potently enhanced the action of forskolin in both parameters of synaptic transmission. The latter could be due (1) to the inhibitory effect of IBMX on phosphodiesterases, (2) to a removal of an inhibition of adenylate cyclase by the tonic activation of $A_{1}$ receptors, and/or (3) to the following effect: the rise in intracellular cAMP levels produced by forskolin application in vitro and in vivo is often accompanied by a rise in extracellular cAMP and its metabolites due to "overflow" of cAMP (Hill, 1985; Knight et al., 1992). The latter activate $\Lambda_{1}$ receptors and would depress synaptic strength (Dunwiddie and Hoffer, 1980). This depressant effect of extracellular cAMP, which is likely to be enhanced following forskolin addition, would be prevented by IBMX.

A negligible contribution of increases in postsynaptic sensitivity to the endogenous neurotransmitter glutamate was ob- served in this study. This is in contrast to the observations of Greengard et al. (1991) and Wang et al. (1991), who found an increase of glutamate-evoked responses in cultured hippocampal neurons. We observed an increase in mEPSC amplitude in $40 \%$ of cells tested, compared to $64 \%$ reported by Greengard et al. (1991). Differences in experimental protocols may contribute. IBMX was not used in conjunction with forskolin in cultured hippocampal neurons (Greengard et al., 1991; Wang et al., 1991). A plausible explanation for our observations is that IBMX may occlude the action of forskolin in postsynaptic cells. However, this is not supported by the observation that IBMX does not alter the amplitude of mEPSCs despite its effect of eEPSCs (Prince and Stevens, 1992). It is also possible that a potentiation of postsynaptic sensitivity can only be revealed in the absence of ATP in the recording pipette (which may, in principle, maintain a high "baseline" PKA activity). However, we do not favor this possibility, since inclusion of $\mathrm{Mg \Lambda TP}$ in the patch solution did not prevent (occlude) the potentiation of mEPSCs upon forskolin application in cultured hippocampal neurons (Greengard et al., 1991).

It is likely that the action of different extracellular modulators that affect adenylate cyclase [such as glutamate, adenosine, noradrenaline, GABA, vasoactive intestinal peptide (VIP); see below] and regulate endogenous PKA activity determine the net effect of activators such as forskolin. Our lack of a significant postsynaptic effect may be due to differences in the concentration and distribution of these modulators in the slice preparation, as opposed to the neuronal cell culture. For example, the inhibition of forskolin-stimulated cAMP formation by metabotropic glutamate receptor (mGluR) activation in the hippocampus appears to be developmentally regulated: this effect is preferentially seen in slices from mature ( $\geq 2$ weeks) rats (Casabona et al., 1992). We worked with slices from $\sim 2-3$-week-old rats; Greengard et al. (1991), with up to 1-week-old pyramidal cells in culture; Wang et al. (1991), with fetal mice neurons grown $7-14 \mathrm{~d}$ in culture. Endogenous extracellular glutamate (Sah et al., 1989) may exert a larger inhibition of adenylate cyclase in our slices than in the cultures, reducing any detectable postsynaptic action of forskolin.

The facilitating action of cAMP on transmitter release could be at a level ranging from mechanisms regulating intraterminal free calcium availability to the sequence of events leading to exocytosis. cAMP has been shown to increase currents through high-threshold voltage-dependent $\mathrm{Ca}^{2+}$ channels (Chetkovich et al., 1991) and to suppress a $\mathrm{Ca}^{2+}$-activated slow $\mathrm{K}^{+}$conductance ( $I_{\mathrm{AHP}}$; Madison and Nicoll, 1982) in CAl hippocampal pyramidal neurons. The presence of either or both of these currents in terminals of CA3 pyramidal cells could enhance depolarization-induced $\mathrm{Ca}^{2+}$ influx and transmitter release. Since the spontaneous release of transmitter was also potentiated by forskolin, an action on another PKA target must also be involved in the action of this diterpene. Phosphorylation of synapsin I (a vesicleassociated protein) by PKA increases the ability of synaptic vesicles to move to the plasma membrane and enhance transmitter release in the squid giant synapse (Llinas et al., 1985); a cAMP-mediated increase in phosphorylation of synapsin I has been reported in the rodent hippocampus (Browning et al., 1990). Another potential target is a synaptic vesicle-associated phosphoprotein (SVAPP-120), which is also phosphorylated by PKA (Greengard et al., 1993).

The modulation of synaptic transmission by forskolin in the hippocampus is not unique to excitatory afferents to field $\mathrm{CAl}$; 
it has also been observed in field CA3 (Hopkins and Johnston, 1988) and the dentate gyrus (Stanton and Sarvey, 1985b). However, the site or mechanism of action of this diterpene has not been investigated. Activation of the adenylate cyclase/PKA signal transduction pathway enhances neurotransmitter release at vertebrate peripheral synapses and in invertebrates. In Aplysia, sensitization is mediated by an increase in transmitter release induced by the elevation of intracellular cAMP in the presynaptic terminals of sensory neurons (Kandel and Schwartz, 1982). The Drosophila mutant dunce, deficient in the cAMP phosphodiesterase (which leads to higher levels of cAMP-dependent phosphorylation in nervous tissues), has been found to display significantly larger end-plate potentials (EPPs) and fewer failures in transmission at the larval neuromuscular junction (NMJ) than wild-type larvae, without a significant change in miniature EPPs (mEPPs), suggesting that the main effect of this mutation is on presynaptic release mechanisms (Zhong and $\mathrm{Wu}, 1991$ ). A role for phosphatases (dephosphorylating enzymes) in presynaptic function has also been reported. In frog, crayfish, and lobster NMJs, the phosphatase inhibitor okadaic acid enhances EPPs by a presynaptic mechanism: mEPP amplitudes are unaffected, whereas mEPP frequency is enhanced (Abdul-Ghani et al., 1991; Swain et al., 1991). Swain et al. (1992) have recently shown that the protein kinase involved in the effect caused by hyperphosphorylation by okadaic acid at frog and crayfish NMJs is PKA.

In the rodent CNS, a presynaptic neuromodulatory role for cAMP has recently been proposed to underlie the effect of glucagon, which enhances glutamatergic excitatory transmission in the ventral hypothalamus (Shoji et al., 1992).

What endogenous transmitters/modulators regulate intracellular cAMP levels in the hippocampus? Activation of mGluRs can engage several signal transduction pathways, including an inhibition of cAMP accumulation (mGluR2, mGluR3, and mGluR4: Tanabe et al., 1992; Schoepp and Conn, 1993). In field CA 1 , activation of $\mathrm{mGluRs}$ depresses excitatory synaptic transmission via a presynaptic site of action (Baskys and Malen$\mathrm{ka}, 1991$ ); the second messenger involved, however, has not been established. A presynaptic autoregulatory mechanism via the activation of mGluks and the engagement of the cAMP cascade may underlie this effect. On the other hand, adenosine receptors, which have becn shown to be tonically activated in hippocampal slices (Dunwiddie and Hoffer, 1980), are coupled to adenylate cyclase (Fredholm et al., 1983). Activation of presynaptic adenosine receptors potently inhibits excitatory neurotransmission in the hippocampus (Prince and Stevens, 1992; Thompson et al., 1992), but a consensus on the signal transduction involved has not been established. Since $A_{1}$ receptors appear to be involved in this depressant effect (Dunwiddie and Fredholm, 1989), it is tempting to suggest that these receptors in presynaptic terminals may be negatively coupled to adenylate cyclase (postsynaptic adenosine receptors do not appear to be coupled to adenylate cyclase; Trussell and Jackson, 1987). A presynaptic inhibitory effect on transmitter release has been observed upon activation of $\mathrm{GABA}_{\mathrm{B}}$ receptors at excitatory hippocampal synapses (Scanziani et al., 1992). In the rodent brain, $\mathrm{GABA}_{\mathrm{B}}$ receptors are also coupled to adenylate cyclase (Hill, 1985; Bowery et al., 1990). $\beta$ - $\Lambda$ drenergic, histamine $\mathrm{H}_{2}$, dopamine D, (Dolphin et al., 1979; Segal et al., 1981), and VIP (Etgen and Browning, 1983) receptors in the hippocampus activate adenylate cyclase. The interaction between these and other endogenous modulators may contribute to the maintenance of cAMP levels in presynaptic terminals, and therefore influence efficacy at excitatory synapses.

Is cAMP, or the activation of adenylate cyclase, involved in LTP? As mentioned in the introductory remarks, an NMDAdependent rise in CAMP levels occurs following LTP induction (Bliss and Collingridge, 1993). Preliminary experiments suggest that a significant occlusion of tetanus-induced LTP, measured as the increase in field EPSP slope, occurs in hippocampal slices in the presence of forskolin/IBMX (L. E. Chavez-Noriega and C. F. Stevens, unpublished observations). Also, Frey et al. (1993) have recently reported a reduction in the late component of LTP caused by a selective PKA inhibitor. More research in this area may provide additional clues as to whether activation of the cAMP cascade participates in the expression of LTP.

In summary, our observations indicate that activation of the cAMP cascade can potentiate synaptic efficacy at central glutamatergic synapses via a presynaptic site of action: an increase in spontaneous and evoked transmitter release appears to be induced by an increase in release probability or in the number of sites available for transmitter release.

\section{References}

Abdul-Ghani M, Kravitz EA, Meiri H, Rahamimoff R (1991) Protcin phosphatase inhibitor okadaic acid enhances transmitter release at neuromuscular junctions. Proc Natl Acad Sci USA 88:1803-1807.

Aghajanian GK, Rasmussen K (1989) Intracellular studies in the facial nucleus illustrating a simple new method for obtaining viable motoneurons in adult rat brain slices. Synapse 3:331-338.

Baskys A, Malenka RC (1991) Agonists at metabotropic glutamate receptors presynaptically inhibit EPSCs in neonatal rat hippocampus. J Physiol (Lond) 444:687-701.

Bekkers JM, Stevens CF (1990) Presynaptic mechanism for long-term potentiation in the hippocampus. Nature 346:724-729.

Bekkers JM, Richerson GB, Stevens CF (1990) Origin of variability in quantal size in cultured hippocampal neurons and hippocampal slices. Proc Natl Acad Sci USA 87:5359-5362.

Bliss TVP, Collingridge GL (1993) A synaptic model of memory: longterm potentiation in the hippocampus. Nature 361:31-39.

Bowery NG, Knott C, Moratalla R, Pratt GD (1990) GABA Geceptors $^{3}$ and their heterogeneity. Adv Biochem Psychopharmacol 46:127-139.

Browning MD, Parfitt KD, Doze VA, Madison DV (1990) Beta-adrenergic receptor activation increases phosphorylation of synapsins I and II and increases synaptic transmission in dentate gyrus but not in area CA1 of the hippocampus. Soc Neurosci Abstr 16:653.

Casabona G, Genazzani AA, Di Stefano M, Sortino MA, Nicoletti F (1992) Developmental changes in the modulation of cyclic AMP formation by the metabotropic glutamate receptor agonist $1 S, 3 R$ aminocyclopentane-1,3-dicarboxylic acid in brain slices. J Neurochem 59:1161-1163.

Chavez-Noriega LE, Stevens CF (1992a) Modulation of synaptic efficacy in field CAl of the rat hippocampus by forskolin. Brain Res 574:85-92.

Chavez-Noriega LE, Stevens CF (1992b) Excitatory postsynaptic currents evoked in CAl hippocampal pyramidal neurons are enhanced by direct activation of adenylate cyclase. Soc Neurosci Abstr 18:640.

Chetkovich DM, Gray R, Johnston D, Sweatt JD (1991) NMDAreceptor activation increases cAMP levels and voltage-gated $\mathrm{Ca}^{2+}$ channel activity in area CA1 of hippocampus. Proc Natl Acad Sci USA 88:6467-6471.

Daly JW, Padgett W, Seamon KB (1982) Activation of cyclic AMPgenerating systems in brain membranes and slices by the diterpene forskolin: augmentation of receptor-mediated responses. J Neurochem 38:532-544.

Del Castillo J, Katz B (1954) Quantal components of the end-plate potential. J Physiol (Lond) 124:560-573.

Dolphin A, Hamont M, Bockaert J (1979) The resolution of dopamine and beta 1 and beta 2 adrenergic-sensitive adenylate cyclase activities in homogenates of cat cerebellum, hippocampus and cerebral cortex. Brain Res 179:305-317.

Dunwiddie TV, Fredholm BB (1989) Adenosine A, receptors inhibit 
adenylate cyclase activity and neurotransmitter release and hyperpolarize pyramidal neurons in rat hippocampus. J Pharmacol Exp Ther 249:31-37.

Dunwiddie TV, Hoffer BJ (1980) Adenine nucleotides and synaptic transmission in the in vitro rat hippocampus. $\mathrm{Br} \mathrm{J}$ Pharmacol 69:5968.

Etgen AM, Browning ET (1983) Activators of cyclic adenosine $3 ' 5^{\prime}$ monophosphate accumulation in rat hippocampal slices: action of vasoactive intestinal peptide (VIP). J Neurosci 3:2487-2493.

Fredholm BB, Jonzon B, Lindstrom K (1983) Adenosine receptor mediated increases and decreases in cyclic AMP in hippocampal slices treated with forskolin. Acta Physiol Scand 117:461-463.

Frey U, Huang Y-Y, Kandel ER (1993) Effects of cAMP simulate a late stage of LTP in hippocampal CA1 neurons. Science 260:16611664.

Greengard P, Jen J, Nairn AC, Stevens CF (1991) Cyclic AMP-dependent protein kinase enhances glutamate response in cultured rat hippocampal neurons. Science 253:1135-1138.

Greengard P, Valtorta F, Czernik AJ, Benfenati F (1993) Synaptic vesicle phosphoproteins and regulation of synaptic function. Science 259:780-785

Haslam RJ, Davidson MML, Desjardins JV (1978) Inhibition of adenylate cyclase by adenosine analogues in preparations of broken and intact human platelets. Biochem J 176:83-95.

Hidaka H, Inagaki M, Kawamoto S, Sasaki Y (1984) Isoquinolinesulfonamides, novel and potent inhibitors of cyclic nucleotide dependent protein kinase and protein kinase C. Biochemistry 23:50365041.

Hill DR (1985) GABA $_{\mathrm{B}}$ receptor modulation of adenylate cyclase activity in rat brain slices. Br J Pharmacol 84:249-257.

Hopkins WF, Johnston D (1988) Noradrenergic enhancement of longterm potentiation at mossy fiber synapses in the hippocampus. J Neurophysiol 59:667-687.

Hoshi T, Garber SS, Aldrich RW (1988) Effect of forskolin on voltagegated $\mathrm{K}^{+}$channels is independent of adenylate cyclase activation. Science 240:1652-1655.

Kandel FR Schwart7. JH (1982) Molecular biology of learning: modulation of transmitter release. Science 218:433-443.

Katz B (1969) The release of neural transmitter substances. Liverpool: Liverpool UP.

Knight AR, Whitton P, Bowery NG (1992) In vitro and in vivo effects of baclofen on cAMP overflow in rat cerebral cortex. Soc Neurosci Abstr 18:1157.

Laurenza A, Sutkowski EM, Seamon KB (1989) Forskolin: a specific stimulator of adenylyl cyclase or a diterpene with multiple sites of action? Trends Pharmacol Sci 10:442-447.

Llinas R, McGuinness TL, Leonard CS, Sugimori M, Greengard P (1985) Intraterminal injection of synapsin I or calcium/calmodulindependent protein kinase II alters neurotransmitter release at the squid giant synapse. Proc Natl Acad Sci USA 82:3035-3039.

Madison DV, Nicoll RA (1982) Noradrenaline blocks accommodation of pyramidal cell discharge in the hippocampus. Nature 299:636-638.

Malinow R, Tsien RW (1990) Presynaptic enhancement shown by whole-cell recordings of long-term potentiation in hippocampal slices. Nature 346:177-180.

Manabe T, Renner P, Nicoll RA (1992) Postsynaptic contribution to long-term potentiation revealed by the analysis of miniature synaptic currents. Nature 355:50-55.

Onodera H, Kogure K (1989) Mapping second messenger systems in the rat hippocampus after transient forebrain ischemia: in vitro $\left[{ }^{3} \mathrm{H}\right]$ forskolin and $\left[{ }^{3} \mathrm{H}\right.$ ]inositol 1,4,5-trisphosphate binding. Brain Res 487:343-349.
Phillis JW, Wu PII (1981) The role of adenosine and its nuclcotides in central synaptic transmission. Prog Neurobiol 16:187-239.

Prince DA, Stevens CF (1992) Adenosine decreases neurotransmitter release at central synapses. Proc Natl Acad Sci USA 89:8586-8590.

Raastad M, Storm JF, Andersen P (1992) Putative single quantum and single fibre excitatory postsynaptic currents show similar amplitude range and variability in rat hippocampal slices. Eur J Neurosci 4:113-117.

Sah P, Hestrin S, Nicoll RA (1989) Tonic activation of NMDA receptors by ambient glutamate enhances excitability of neurons. Science 246:815-818.

Scanziani M, Capogna M, Gahwiler BH, Thompson SM (1992) Presynaptic inhibition of miniature excitatory synaptic currents by baclofen and adenosine in the hippocampus. Neuron 9:919-927.

Schoepp DD, Conn PJ (1993) Mctabotopic glutamatc receptors in brain function and pathology. Trends Pharmacol Sci 14:13-20.

Seamon KB, Daly JW (1986) Forskolin: its biological and chemical properties. Adv Cyclic Nucleotide Protein Phosphorylation Res 20: $1-147$.

Segal M, Greenberger V, Hofstein R (1981) Cyclic AMP-generating systems in rat hippocampal slices. Brain Res 213:351-364.

Shoji S, Akasu T, Nonaka K (1992) Presynaptic facilitation of excitatory postsynaptic potential by glucagon in neurons of rat ventromedial hypothalamic slices. Neurosci Lett 138:291-294.

Smellie FW, Davis CW, Daly JW, Wells JN (1979) Alkylxanthines: inhibition of adenosine-elicited accumulation of cyclic AMP in brain slices and of brain phosphodiesterase activity. Life Sci 24:2475-2482.

Stanton PK, Sarvey JM (1985a) The effect of high-frequency electrical stimulation and norcpincphrinc on cyclic AMP levels in normal versus norepinephrine-depleted rat hippocampal slices. Brain Res 358: 343-348.

Stanton PK, Sarvey JM (1985b) Blockade of norepinephrine-induced long-lasting potentiation in the hippocampal dentate gyrus by an inhibitor of protein synthesis. Brain Res 361:276-283.

Swain JE, Robitaille R, Dass GR, Charlton MP (1991) Phosphatases modulate transmission and serotonin facilitation at synapses: studies with the inhibitor okadaic acid. J Neurobiol 22:855-864.

Swain JE, Robitaille R, Charlton MP (1992) Phosphatase inhibitors interact with protein kinases to regulate neurotransmitter release but do not affect $\mathrm{Ca}^{2+}$ homeostasis. Soc Neurosci Abstr 18:635.

Tanabe Y, Masu M, Ishii T, Shigemoto R, Nakanishi S (1992) A family of metabotropic glutamate receptors. Neuron 8:169-179.

Thompson SM, Ilaas IIL, Gahwiler BH (1992) Comparison of the actions of adenosine at pre- and postsynaptic receptors in the rat hippocampus in vitro. J Physiol (Lond) 451:347-363.

Trussell LO, Jackson MB (1987) Dependence of an adenosine-activated potassium current on a GTP-binding protein in mammalian central neurons. J Neurosci 7:3306-3316.

Wagoner PK, Pallotta BS (1988) Modulation of acetylcholine receptor desensitization by forskolin is independent of cAMP. Science 240 : 1655-1657.

Wang L-Y, Salter MW, MacDonald JF (1991) Regulation of kainate receptors by cAMP-dependent protein kinase and phosphatases. Science 253:1 132-1135.

Wang L-Y, Taverna FA, Huang X-P, MacDonald JF, Hampson DR (1993) Phosphorylation and modulation of a kainate receptor (GluR6) by cAMP-dependent protcin kinasc. Science 259:1173-1175.

Zhong Y, Wu C-F (1991) Altered synaptic plasticity in Drosophila memory mutants with a defective cyclic AMP cascade. Science 251: 198-201. 